\title{
The Sol-Gel Process For Realisation Of Optical Micro-Structures in Glass
}

\author{
V.K. Parashar, A.Sayah and M.A.M. Gijs \\ Institute of Microelectronics and Microsystems
}

Swiss Federal Institute of Technology Lausanne, $\mathrm{CH}-1015$ Lausanne EPFL, Switzerland

\section{Keywords: Microfabrication, Sol-Gel, Diffractive Optical Elements, Microlens}

\begin{abstract}
We have applied the sol-gel process to generate optical microstructures in glass using in-house developed sol-gel materials. The synthesis of our sol-gel glass materials is based on the formation of particles of controlled size from organo-metallic compounds. The overall shrinkage during annealing of the gel material is negligible and does not imply crack formation. Silica, titania and binary oxides of silica titania oxide sol-gel materials have been used. A two-step pattern transfer is employed to replicate the structure in glass: first a polydimethylsiloxane (PDMS) replica is obtained from a $\mathrm{Si}$, polymethylmethacrylate (PMMA) or polyimide (PI) master structure, and secondly, a layer of sol-gel material is applied on the PDMS 'soft-replica' to get diffractive micro-/nano-structures in glass after drying and annealing. These structures are characterised by a scanning electron microscope (SEM) and by an optical diffraction setup.
\end{abstract}

\section{Introduction}

Sub-micrometer structures, particularly optical elements, are necessary in advanced microopto-electro-mechanical systems (MOEMS) and, therefore represent a research area of great importance. Such elements can today be fabricated through the use of expensive processes, like ion etching, e-beam/laser writing, which are limited in performance [1,2]. For example, it is difficult to obtain a smooth concave, convex or prismatic profile using these techniques; on the other hand, polymer/plastic materials are easily microfabricated using molding techniques and are currently in use for micro-optical applications [3]. However, for certain applications, plastic structures present problems, for example in those where optical components are exposed to strong irradiation, giving rise to degradation of the plastic polymer chains, or in applications where temperature of operation is strongly varying, so that incompatibilities between dilatation of plastic structures and glass parts lead to a deterioration of the optical device functioning. Therefore, an attractive option is to develop a molding technology in glass. However, the mechano-chemical inertness of glass makes it hard for microstructuring. The only possible way to replicate glass is the direct casting from a liquid solution. Melt molding of glass is not a feasible solution, but chemical processing of a glass using liquid precursors provides a better opportunity to microstructure glass components with desired physico-optical properties. This process, known as the sol-gel process, can be easily modified to prepare a desired glass network. We have realized arrays of glass micro-optical elements like microlenses, channel gratings, holographic gratings, fresnel lenses using in-house developed sol-gel materials [4,5].

\section{Experimental}

Two classes of sol-gel materials are prepared: (i) polymeric and (ii) nano-colloidal materials. The principal oxides used are silicon oxide, titanium oxide, and their binary oxides. Polymeric sol-gel materials consist of sub-micron size linear chains of an oxide network with reactive hydroxyl groups. Nano-colloidal sol gel materials are composed of nano-sized metal 


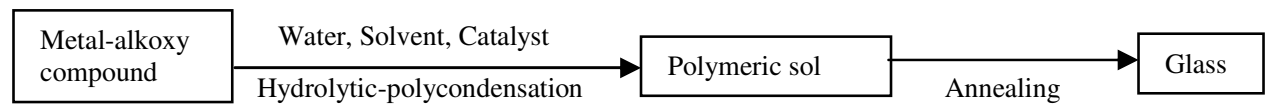

Figure 1a : Schematic of Polymeric sol-gel material formation

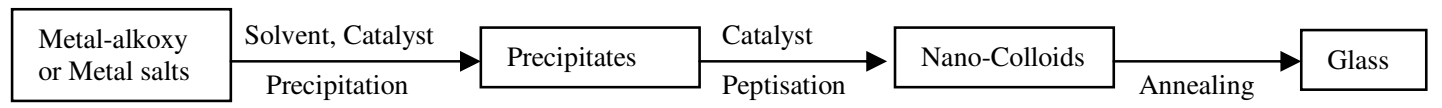

Figure 1b : Schematic of nanocolloidal sol-gel material formation

oxide stabilized in an appropriate solvent. Figure 1 shows a schematic diagram of the sol formation.

Figure 2 describes the fabrication sequence for arrays of glass micro- and nano-structures. The fabrication process involves two main steps. The first step is the transfer of a master microstructure to a PDMS film. PDMS is spin-coated on the patterned silicon wafer at a speed of 400 and $450 \mathrm{rpm}$ for $15 \mathrm{~s}$ each and dried under vacuum for 30-60 min. Then a heat cure is carried out at $60{ }^{\circ} \mathrm{C}$ for $24 \mathrm{~h}$. Subsequently, we gently peel away the PDMS replica from the silicon wafer.

The glass microstructure is obtained from the PDMS replica to the sol-gel film as shown in figure 2, which on further heat treatment provides a glass optical structure with micro-/nanoscale features. Dried gel structures are further heat treated to $600{ }^{\circ} \mathrm{C}$ at an appropriate heating rate under ambient atmospheric conditions for about $100 \mathrm{~h}$ to get a pure glass micro-/nanostructure.

\section{Results and Discussion}

Figure 3 shows SEM photographs of various optical elements having micro-scale features. Columnar grating structures show a high quality surface having a very low surface roughness in the range of few nanometres. These gratings show perfect structural replication from the master structure.

The diffraction efficiency of the gratings is evaluated using a He-Ne laser $(633 \mathrm{~nm})$. The measurements were performed in reflection and transmission modes for the gratings formed in glass, as shown in figure 4 . The laser irradiation shows high order diffraction spots, which is a proof of the quality of our glass micro- structures.

(a)

Si microstructure

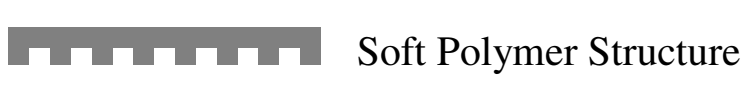

(b)

Sol gel material coated on

Soft Polymer

(c) Optical structure after annealing

Figure 2 : Fabrication sequence of for replication of optical micro-structures (a) Preparation of a soft polymer structure from a silicon microstructure; (b) Coating of the sol gel material; (c) Production of an optical structure by annealing of the sol gel material. 

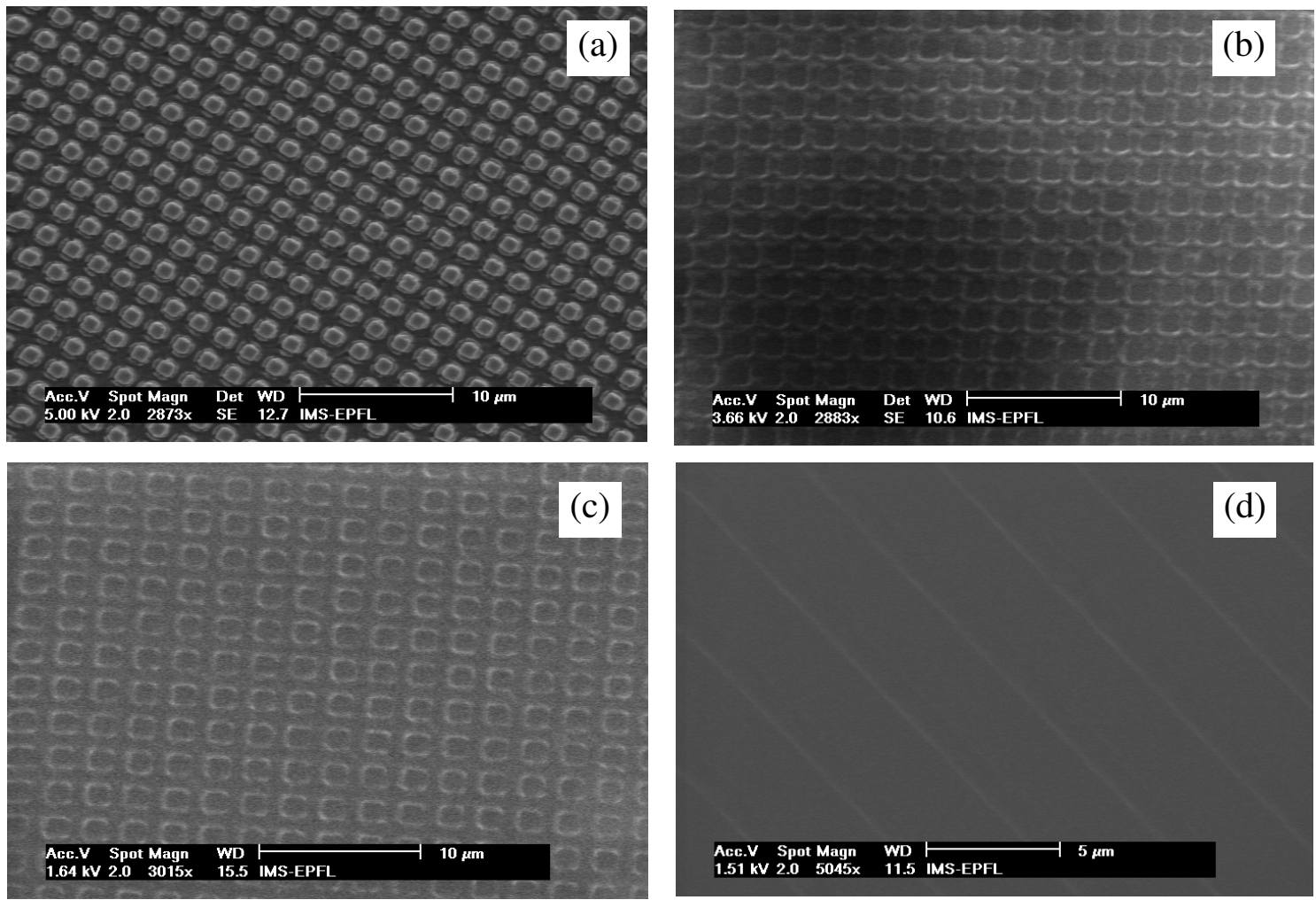

Figure 3 : Scanning electron micrographs of (a) columnar grating structure in silicon prepared by reactive ion etching in a $\mathrm{SF}_{6} / \mathrm{O}_{2}$ plasma to a depths of $800 \mathrm{~nm}$; (b) negative replica of columnar grating structure in PDMS film using the above described procedure; (c) columnar grating structure replicated in sol-gel derived silica glass and heat treated to $600{ }^{\circ} \mathrm{C}$; (d) channel grating structure replicated in sol-gel derived silica glass and heat treated to $600{ }^{\circ} \mathrm{C}$.
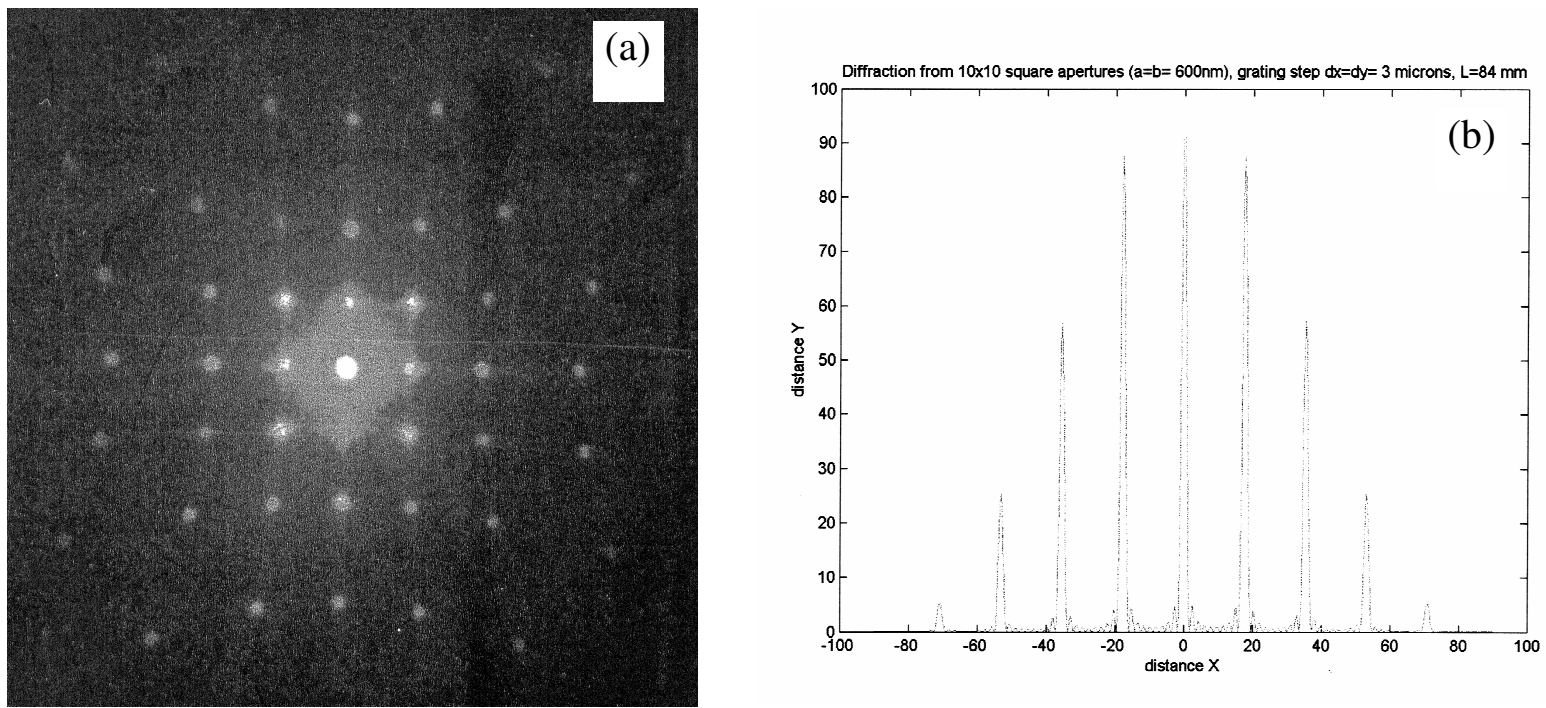

Figure 4: (a) Diffraction pattern of a columnar grating structure; (b) calculated intensity profile of diffraction of the given grating. 


\section{Conclusion}

The effectiveness of sol-gel derived materials for glass microstructuring has been demonstrated. Our work offers great potential for low-cost and large-scale manufacturing of sol-gel integrated optic devices employing optical elements in the micro- and nano-metre domain.

\section{References}

[1] X. Li, T. Abe and M. Eshashi : Sens \& Act. A. 87 (2001), p. 139.

[2] C. David, J. Wei, T. Lippert and A. Wokaun : Microelec. Eng. 57-58 (2001), p. 453.

[3] M.T. Gale : Microelect. Eng. 34 (1997), p. 321.

[4] A. Sayah, V.K. Parashar and M.A.M. Gijs : Proceed. IEEE-MEMS 2002 Conference, Las Vegas, USA, January 20 - 24, 2002, p. 516.

[5] V.K. Parashar, A. Sayah, M. Pfeffer, F. Schoch, J. Gobrecht and M.A.M. Gijs : Proceed. MNE-02 Conference, Lugano, Switzerland, Sept. 16-19, 2002, p. 250. 